\title{
Nouvelles technologies de production : comment utiliser la méthode de la chaîne critique pour optimiser les délais et l'allocation des ressources dans les projets
}

\author{
Serge Nenkam, Docteur en management de projets \\ Caroline Gagné, Ph.D., professeure \\ Université du Québec à Chicoutimi
}

\section{INTRODUCTION}

Il est question de présenter la théorie des contraintes du Professeur Goldratt qui a donné naissance à la méthode de la chaîne critique.

L'entreprise peut être présentée comme un système d'activités humaines constitué d'un ensemble cohérent et autonome d'interactions capables de transformer des éléments en entrée (inputs) en d'autres éléments de sortie (outputs) qui apportent une valeur ajoutée aux intrants. Dans le but de réaliser son plan stratégique, l'entreprise conçoit et met en œuvre des initiatives temporaires devant aboutir à la création d'un produit, d'un service ou d'un résultat unique qui répond tant aux besoins de ses clients qu'aux exigences liées à sa capacité de production (coûts, ressources humaines, machines, espace). Ces initiatives, qui favorisent à la fois l'innovation et la créativité, sont des projets (Kerzner, 2013). Dans ce but, grâce aux projets, l'entreprise produit sur le marché des biens et/ou des services destinés à la satisfaction des clients, contribuant ainsi à l'augmentation de ses différentes ressources productrices.
Afin de toujours optimiser son rendement et résister à la concurrence, l'entreprise doit tendre en permanence vers l'excellence par le biais des améliorations continues. Ce challenge se manifeste aussi souvent par la recherche perpétuelle de nouvelles technologies de production capables d'anticiper les retards dans les projets. Dans la littérature, la chaîne critique, développée par le professeur Elliot Goldratt), est souvent considérée comme une nouvelle technologie de production dont les effets sur les projets sont probants et les résultats satisfaisants. Cependant, son implantation est souvent problématique et nécessite beaucoup de pédagogie.

Dans cet article, il est question de présenter la théorie des contraintes du Professeur Goldratt qui a donné naissance à la méthode de la chaîne critique. Ensuite, à partir d'un cas réel de problématique organisationnelle, nous montrons comment cette technologie de production est encore d'actualité aujourd'hui et permet de répondre efficacement à la problématique d'optimisation des délais et d'allocation des ressources dans les projets.

\section{PRÉSENTATION DE LA THÉORIE DES CONTRAINTES (THEORY OF CONSTRAINTS - TOC)}

Les bases de la théorie des contraintes ont été introduites dans le courant des années 1980 par Goldratt (1984) dans son ouvrage intitulé The goal. Toutefois, c'est en 1990 que la théorie des contraintes s'est révélée comme un véritable paradigme en management lorsque Goldratt (1990) a affirmé que mettre l'accent sur le coût du produit ne peut pas promouvoir la performance globale d'une entreprise. La théorie des contraintes suppose que chaque organisation fait face à au moins une contrainte dans un cycle de production. Elle met l'accent sur l'amélioration des ventes par la gestion optimale des goulots d'étranglement dans l'ensemble du système de production. Dans sa conception philosophique, les prémisses de la théorie des contraintes se basent sur le fait que l'objectif d'une entreprise est de faire plus d'argent maintenant et dans l'avenir (Goldratt 1990, p. 12). La théorie des contraintes accorde une importance capitale aux ventes, plutôt qu'aux inventaires de stocks de produits fabriqués. 
Selon Goldratt (1992), une contrainte est tout élément ou facteur qui limite le système de faire ce pour quoi il a été conçu. Plusieurs études ont montré que l'application de la théorie des contraintes au sein des organisations permet d'améliorer considérablement la rentabilité des organisations (Mabin et Balderstone, 1998). La théorie des contraintes encourage les gestionnaires au sein des organisations à identifier le plus tôt possible les éléments qui peuvent limiter les options de réalisation des projets et à anticiper des solutions potentielles. Elle intervient plus particulièrement dans trois domaines précis : la logistique, la mesure des performances et la pensée logique (Cox et Spencer, 1998; Simatupang et al., 2004). Appliquée à la logistique, la théorie des contraintes permet de mettre en œuvre un séquencement adéquat des processus. Pour la mesure des performances, la théorie des contraintes prévoit la mesure des inventaires et des dépenses opérationnelles. Dans la pensée logique, la théorie des contraintes détermine les cinq étapes nécessaires à la gestion des contraintes :
1. Identifier les contraintes du système

2. Prendre des options pour exploiter les contraintes du système

3. Subordonner toute action aux résultats obtenus de l'exploitation des contraintes

4. Lever les contraintes du système et

5. Reprendre à la première étape

La théorie des contraintes s'est successivement développée dans le marketing (Goldratt, 1994) puis dans le management de projet (Goldratt, 1997). Elle permet en particulier la réduction des marges libres et totales, des temps de cycle et l'amélioration significative des performances des délais et des coûts des projets.

\section{Plusieurs études ont montré que l'application de la théorie des contraintes au sein des organisations permet d'améliorer considérablement la rentabilité des organisations.}

\section{DESCRIPTION DE LA MÉTHODE DU MANAGEMENT DE LA CHAÎNE CRITIQUE}

\section{La théorie des contraintes vise à favoriser l'efficacité des équipes de projet, à éviter les goulots d'étranglement et à stimuler la réalisation des projets dans des délais plus courts.}

La chaîne critique est une méthode de management qui a été développée et mise au point par Goldratt (1997) et publiée dans son livre « Critical Chain ». Elle est le prolongement et l'application même de la théorie des contraintes dans le management des projets. Kendall et Austin (2013) affirment d'après leur étude que l'utilisation de la chaîne critique dans la gestion des projets a montré sa capacité à réduire fortement les gaspillages de temps, de ressources et à améliorer le contrôle des coûts. Ces deux auteurs ont listé un ensemble de 64 entreprises de secteurs divers qui ont fait l'expérience de cette démarche et dont les résultats obtenus ont tous été satisfaisants. Parmi les résultats les plus frappant de l'étude de Kendall et Austin (2013), certains attirent l'attention: dans le secteur de l'aérospatial par exemple, l'entreprise Lord Corporation mentionne une amélioration de $60 \%$ de sa capacité de production sans aucun licenciement ni recrutement. Boeing (dans le domaine militaire) relève une réduction de plus de $50 \%$ du temps requis pour l'assemblage des ailes d'avion. Dans l'industrie de l'aluminium, l'entreprise Alcan Alesa Technologies parle d'une augmentation de $30 \%$ du nombre de projets achevés dans les délais. Dans l'industrie de l'automobile, Chrysler note une réduction du temps de cycle de construction des prototypes de 12 à 7 mois. Dans le secteur des communications, le cas le plus frappant est celui de l'entreprise eIRcom qui relève une augmentation de 40 à $90 \%$ des projets terminés à temps avec une réduction des délais de traitement de 150 à 30 jours. Dans l'industrie métallurgique, l'entreprise Tata Steel évoque une amélioration de $68 \%$ de la rapidité d'exécution des projets, avec un temps d'arrêt des activités planifiées qui est passé de 11 jours à 5 jours. Du côté du textile, Skye Group mentionne une réduction de $30 \%$ des délais de traitement avec $100 \%$ des dates de livraison honorées.

La théorie des contraintes vise à favoriser l'efficacité des équipes de projet, à éviter les goulots d'étran- 
glement et à stimuler la réalisation des projets dans des délais plus courts.

Goldratt (1997) énumère les étapes de la mise en œuvre de la planification par la chaîne critique. Dans la pratique, ces étapes peuvent s'articuler de manière itérative. Il s'agit de :

1. Parcourir la chaîne de production pour déterminer la date de fin du projet et utiliser la date de livraison comme la cible à atteindre.
2. Planifier les activités avec la méthode classique du chemin critique (Critical Path Method) en tenant compte de la date de réalisation au plus tard.

3. Changer l'estimation des durées des activités en intégrant la disponibilité des ressources et les contraintes de l'entreprise.

4. Procéder à l'élimination des conflits de ressources pour déterminer la chaîne critique du projet. Cette étape constitue le nivellement des ressources et s'effectue à plusieurs reprises.

5. Ajouter les délais de contingence pour gérer les écarts de temps et de ressource.

\section{CAS PRATIQUE D'IMPLANTATION DE LA CHAÎNE CRITIQUE DANS UN PROJET RÉEL}

\section{1 Énoncé du cas}

L'implantation de la méthode de la chaine critique se déroule dans une entreprise de transformation de l'aluminium installée au Québec. Le tableau 1 présente l'ensemble des activités recensées et codifiées, ainsi que leurs prédécesseurs, leurs durées moyennes et les responsables qui y interviennent. Les durées sont indiquées en semaines. Dans ce cas précis, les noms des ressources sont fictifs pour respecter l'anonymat des employés.

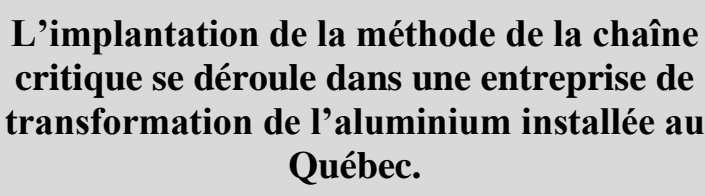

Tableau 1 - Cas d'application de la chaîne critique

\begin{tabular}{|l|c|c|l|l|}
\hline Code & Nom de l'activité & Prédécesseur & Durée & Ressources \\
\hline A & Chargement de la baie & - & 4 & Jean \\
\hline B & Pré-broyage & - & 8 & Paul \\
\hline C & Séparation magnétique & - & 1 & Martin \\
\hline D & Multi-clonage & C & 1 & Paul + Jean \\
\hline E & Tamisage & A & 6 & Martin+Jean \\
\hline F & Séparateur à avalanche & A & 3 & Alain \\
\hline G & Triage & B & 5 & Pierre \\
\hline H & Vérification & E, F, G & 3 & Antoine \\
\hline I & Codification & D & 1 & Martin \\
\hline J & Enregistrement & I & 2 & Paul \\
\hline K & Stockage en sac scellé & H & 2 & Jean \\
\hline L & Préparation de la livraison & J, K & 5 & Philippe \\
\hline
\end{tabular}


Dans ce cas précis, le directeur de production de l'entreprise nous confie que les projets de transformation de cette taille sont réalisés en moyenne en 35 semaines. De plus, la ressource «Alain» prend un congé de quatre semaines pendant sa période de travail. Le directeur de la production prévoit également qu'il est possible que la trieuse que doit utiliser la ressource «Pierre » soit indisponible pendant trois semaines. Ainsi, il est demandé de vérifier la durée prévue du projet et de regarder l'effet de l'indisponibilité des ressources humaines et matérielles sur l'échéancier.

Afin d'apporter une réponse efficace à cette problématique, nous optons pour la mise en œuvre d'une démarche scientifique capable de calculer, dans un premier temps, la durée prévue du projet et d'observer, dans un second temps, l'effet de l'indisponibilité des ressources (humaines et matérielles) sur les délais. Notre démarche, qui s'appuie sur les cinq étapes énoncées par Goldratt (1997) et que nous présentons à la Section 2, sera articulée comme suit :

i- déterminer la durée prévue de réalisation du projet actuel sans tenir compte des ressources

ii- pour chaque activité, déterminer sa marge libre et sa marge totale dans le but d'observer la possibilité d'effectuer ou non des retards

iii- étudier l'effet du congé de la ressource humaine « Alain » qui accuse une absence de quatre semaines pendant la réalisation du projet; et iv- étudier l'effet de l'indisponibilité de la ressource matérielle (trieuse) pendant trois semaines sur l'échéancier et anticiper les prévisions de délais

\subsection{Résolution du cas}

\subsubsection{Calcul de la durée prévue de réalisation du projet}

Pour déterminer la durée réelle du projet, les activités sont d'abord classées par niveau. C'est à dire par ordre de réalisation. Dans cet ordre d'idées, les activités de « Niveau 1 » sont les activités qui n'ont aucun prédécesseur. Les activités de « Niveau 2 » sont précédées par les activités de « Niveau 1 ». Les activités de «Niveau 3 » sont précédées par les activités de « Niveau 2 ». Ainsi, en généralisant, les activités de « Niveau $\mathrm{N} »$ seront précédées des activités de « Niveau $(\mathrm{N}-1)$ ».

Dans notre exemple, les différentes activités du projet se résument en cinq niveaux :

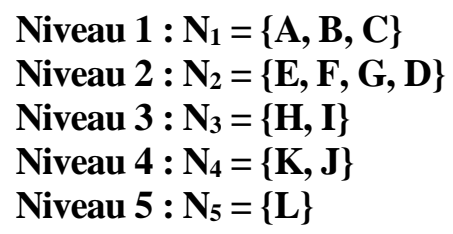

La figure 1 présente le diagramme réseau de ce projet conçu à l'aide de la Méthode des Potentiels Métras (MPM). La méthode MPM est une technique de représentation des graphes orientés inventée par Bernard Roy en 1958 utilisée en gestion de projet (Alexandre, 2003). Elle vise à optimiser la planification des tâches du projet. Une activité est représentée dans une case, avec à gauche sa date de début au plus tôt et à droite sa date de début au plus tard.

Figure 1 - Diagramme réseau du projet et chemin critique

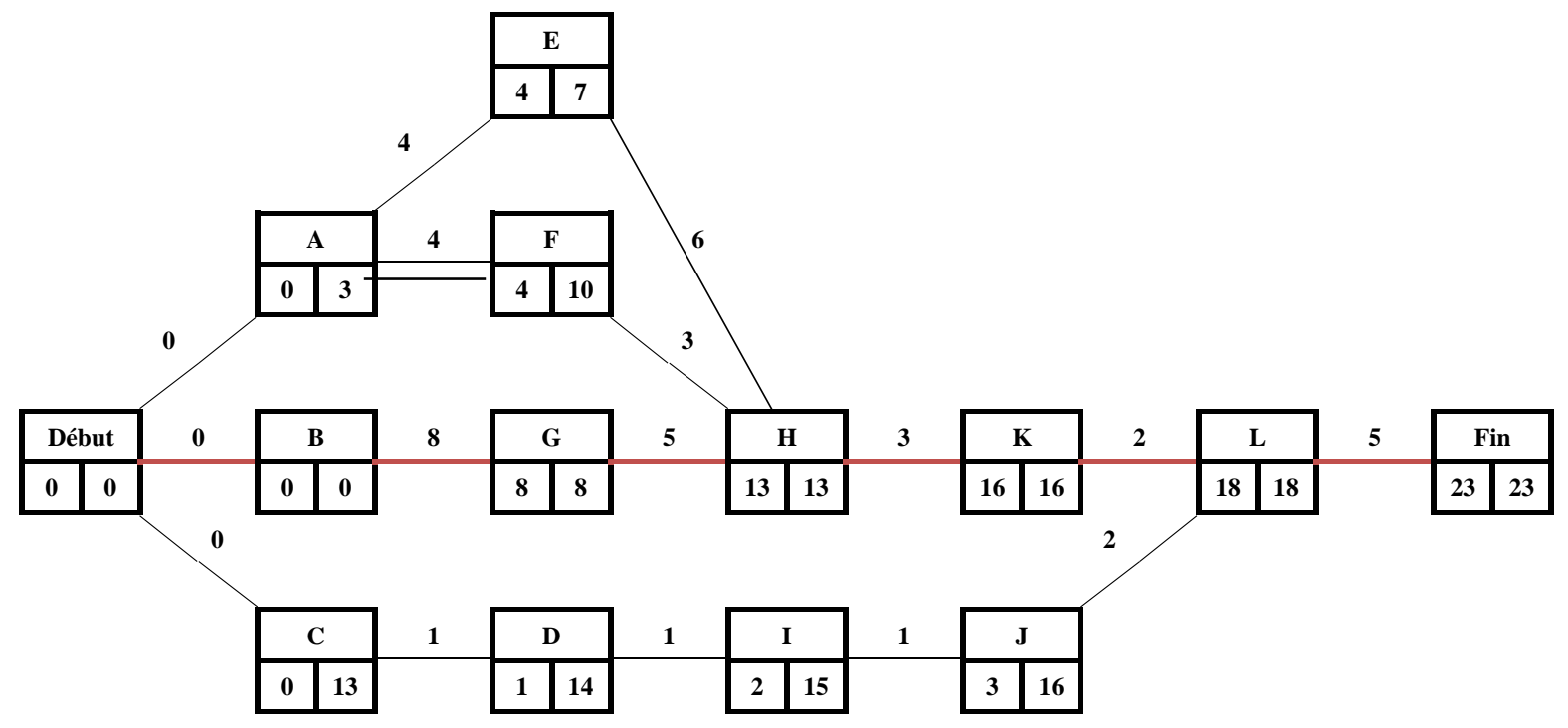


Le chemin critique du projet passe par les activités Début, B, G, H, K, L et Fin. Ce chemin critique est indiquée en rouge sur la figure 1 . La durée de réalisation $\mathrm{du}$ projet est de 23 semaines. Contrairement aux prévisions du directeur de production qui anticipait une durée de réalisation de 35 semaines, nous constatons que la démarche scientifique nous permet de dégager des gaspillages de durée de l'ordre de 12 semaines pour ce projet.

\subsubsection{Pour chaque activité, déterminer la marge} libre et la marge totale

Le tableau 2 présente les marges libres et les marges totales du projet. La marge libre d'une activité est la durée dont on peut décaler sa date de fin sans retarder la date de début au plus tôt des tâches successeurs. La marge totale d'une activité est la durée dont on peut décaler sa date de fin sans retarder la date de fin du projet. Comme on peut le constater sur le tableau 2, les activités situées sur le chemin critique ont une marge libre et une marge totale nulles.

Tableau 2 - Marges libres et totales du projet

\begin{tabular}{|l|c|c|c|c|c|c|c|c|c|c|c|c|}
\hline Désignations Activités & A & B & C & D & E & F & G & H & I & J & K & L \\
\hline Marge Libre (ML) & 0 & 0 & 0 & 0 & 3 & 6 & 0 & 0 & 0 & 13 & 0 & 0 \\
\hline Marge totale (MT) & 3 & 0 & 13 & 13 & 3 & 6 & 0 & 0 & 13 & 13 & 0 & 0 \\
\hline
\end{tabular}

3.2.3. Évaluer l'effet de l'indisponibilité de la ressource humaine "Alain » pendant quatre semaines

Si l'activité $F$ accuse une contrainte de temps d'indisponibilité (4 semaines), la nouvelle durée de F devient :

Durée $F: 3+4$, soit sept semaines

En intégrant cette contrainte au diagramme réseau précédent, on obtient la figure 2 avec les dates modifiées que nous distinguons en orange. Comme on peut le constater, cette absence de quatre semaines accusée par la ressource "Alain » n'a aucun impact sur la durée de réalisation prévue du projet. Au fait, la marge totale de l'activité $\mathrm{F}$ que «Alain » réalise est de six semaines au tableau 2. Par conséquent, tant que la modification apportée sur le chemin de l'activité $F$ ne dépasse pas cette valeur de six semaines, la durée de réalisation prévue du projet ne sera pas affectée.

Figure 2 - Chaîne critique du projet intégrant l'indisponibilité de la ressource « Alain »

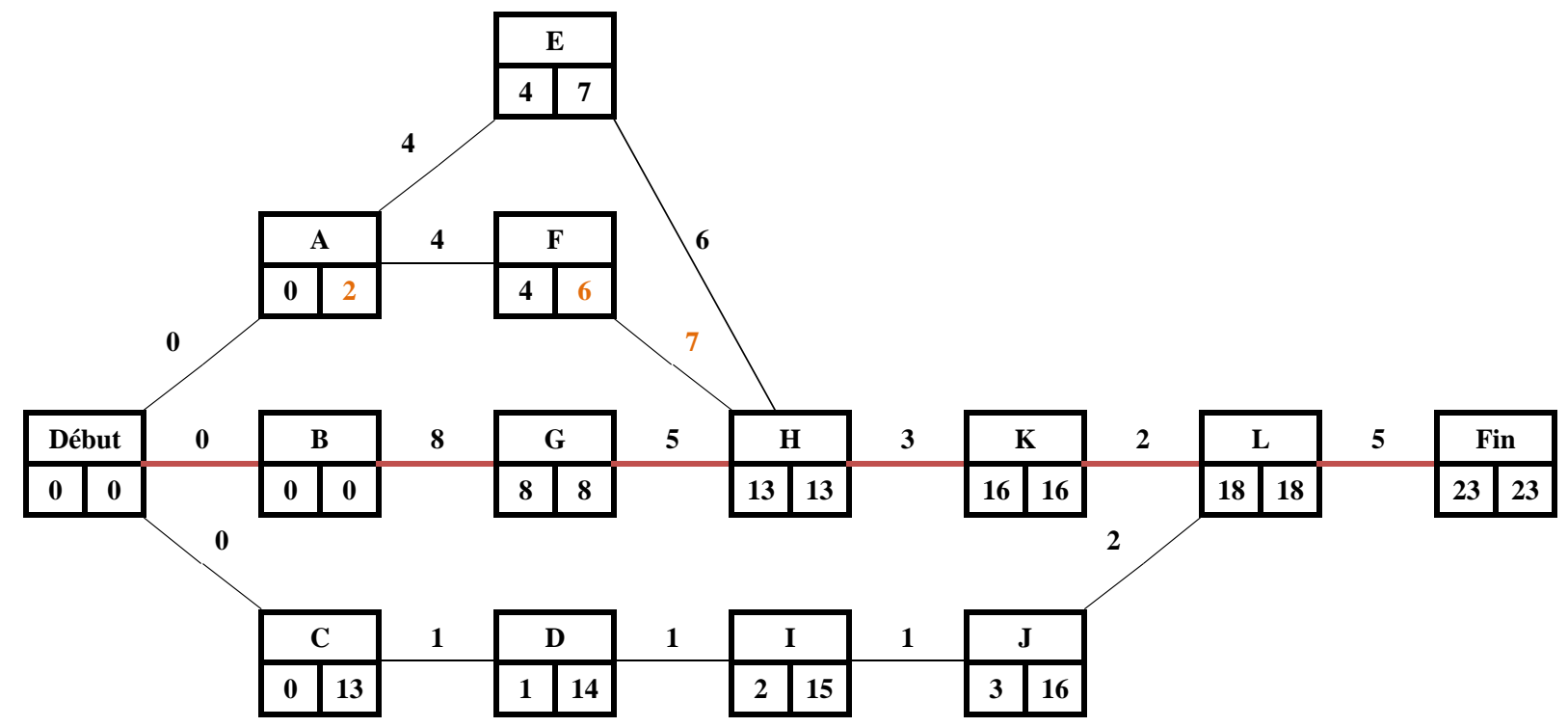




\subsubsection{Analyser l'effet de l'indisponibilité de la ressource matérielle sur le projet}

Si le triage accuse un retard de trois semaines dû à l'attente d'une pièce de rechange, la nouvelle durée pour réaliser cette activité s'allonge à : $G$ : $5+3$, soit huit semaines.

En intégrant cette durée révisée comme contrainte au diagramme réseau de la figure 1 , nous obtenons de nouvelles modifications représentées à la figure 3. Pour marquer l'effet de la modification, les nouvelles dates sont indiquées en bleu. Comme on peut le constater, la durée de réalisation révisée $\mathrm{du}$ projet s'allonge à 26 semaines. Ceci se justifie par le fait que l'activité de triage est située sur le chemin critique qui est, rappelons-le, le chemin le plus long du projet.

Figure 3 - Chaîne critique du projet intégrant l'indisponibilité de la ressource matérielle

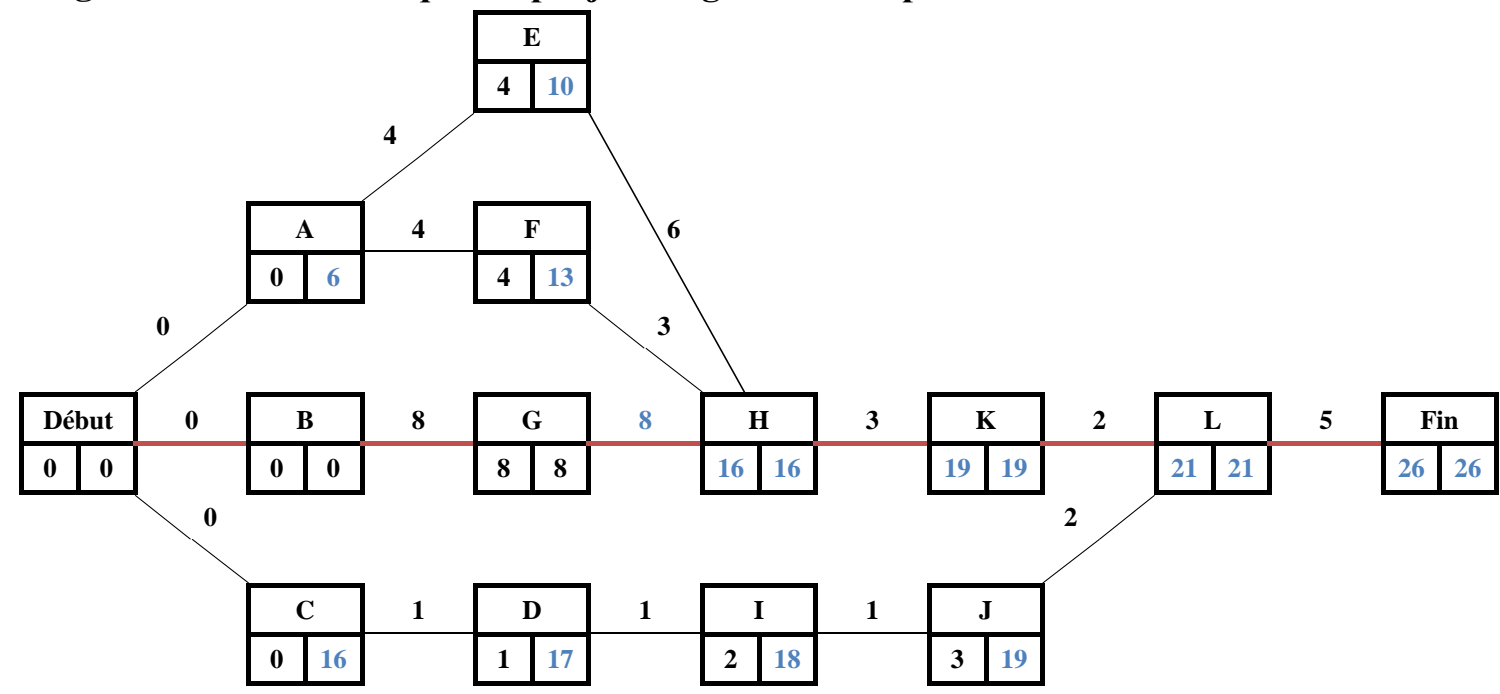

\section{CONCLUSION}

La prise de conscience par la direction de l'entreprise de l'efficacité de l'application de la méthode de la chaîne critique a permis d'obtenir des résultats impressionnants.

Avant le début de l'implantation, la plupart des projets accusaient des retards considérables. La prise de conscience par la direction de l'entreprise de l'efficacité de l'application de la méthode de la chaîne critique a permis d'obtenir des résultats impressionnants.

Quelques temps après l'application de la méthode, les résultats obtenus sont perceptibles et la production a atteint un niveau de réduction des délais dans la réalisation de ses projets que même la direction n'avait pas envisagé. Selon notre expérience, la mise en œuvre de la méthode de la chaîne critique peut apporter des améliorations considérables au sein des projets des entreprises. Elle est encore d'actualité. Plusieurs résultats probants ont été constatés: une amélioration de $37.5 \%$ du temps du cycle de production, avec $75 \%$ d'amélioration des gaspillages des délais et $50 \%$ de récupération des durées de réalisation des projets. Le tableau 3 récapitule les améliorations observées.

Tableau 3 - Résultats de l'application de la planification par la chaîne critique

\begin{tabular}{|l|c|c|c|}
\hline & $\begin{array}{c}\text { Temps de cycle } \\
\text { moyen des projets } \\
(\text { mois })\end{array}$ & $\begin{array}{c}\text { Retard moyen } \\
(\%)\end{array}$ & $\begin{array}{c}\text { Durée moyenne des } \\
\text { projets (mois) }\end{array}$ \\
\hline Avant le MCC & 4 & 40 & 6 \\
\hline Après la chaîne critique (6mois) & 2.5 & 10 & 3 \\
\hline Amélioration & $\begin{array}{c}((4-2.5) / 4) * 100 \\
37.5 \%\end{array}$ & $\begin{array}{c}(\mathbf{4 0 - 1 0}) / 40) * 100 \\
=75 \%\end{array}$ & $((6-3) / 6) * 100=50 \%$ \\
\hline
\end{tabular}




\section{BIBLIOGRAPHIE}

Alexandre, Victor. (2003). Éléments de praxéologie : contribution à une science des actes, Editions L'Harmattan.

Cox, J.F. et Spencer, M. S., (1998). The Constraints Management Handbook, Lucie Press, Boca Raton, FL. Goldratt, E. M., (1984). The Goal: A Process Of Ongoing Improvement.

Goldratt, E. M., (1990). The haystack syndrome, New York: North River Press.

Goldratt, E. M., (1992). From cost world to throughput world, Advances in Management Accounting 1, 3553.

Goldratt, E. M., (1994). It's not luck, Great Barrington, MA: North River Press.

Goldratt, E. M. (1997). Critical Chain, North River Press, Great Barrington.

Kendall, G. I. et Austin, K. M. (2013). « Advanced Multi-Project Management; Achieving Outstanding Speed and Results with Predictability ».

Kerzner, Harold (2013). Project Management: A Systems Approach to Planning, Scheduling, and Controlling, 11th Edition, Wiley.

Mabin, V. and Balderstone, S. (1998). A review of Goldratt's theory of constraints (TOC) - lessons from the international literature. Presentation at the University of Auckland, 16 October 1998.

Simatupang, T. M., Wright, A. C., Sridharan, R., (2004). «Applying the theory of constraints to supply chain collaboration », Supply Chain Management: An International Journal, Vol. 9, Iss 1, pp. 57 - 70. 


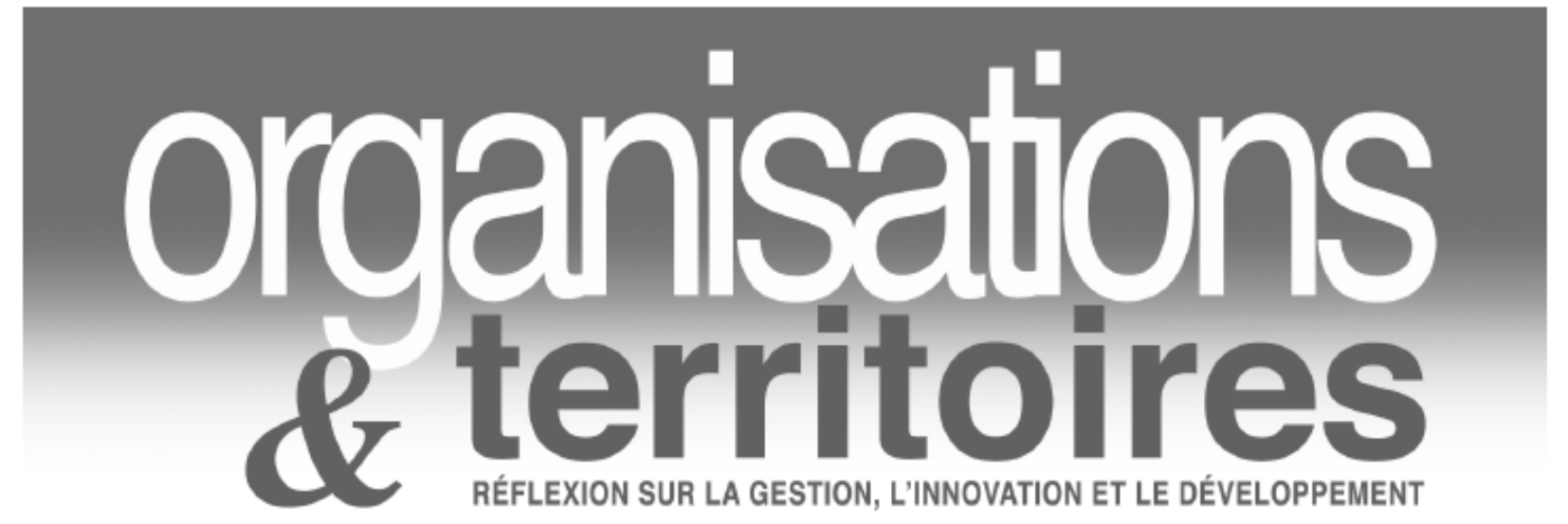

\section{Site Web}

\section{WwW.uqac.ca/revueot \\ www.uqac.ca/revueot \\ www.uqac.ca/revueot \\ www.uqac.ca/revueot}

Nous vous invitons à l'explorer et à nous faire parvenir vos commentaires et suggestions afin d'en améliorer le contenu et la présentation. 